patients in a tertiary-care teaching hospital. A computer screen displayed, at the time of physician order entry, an adaptation of the CDC guidelines for appropriate vancomycin use. The main outcome measures were the frequency of initiation and renewal of vancomycin therapy, as well as the duration of therapy prescribed on a per prescriber basis.

Compared with the control group, intervention physicians wrote $32 \%$ fewer orders (11.3 vs 16.7 orders per physician; $P=.04)$ and had $28 \%$ fewer patients for whom they either initiated or renewed an order for vancomycin (7.4 vs 10.3 orders per physician; $P=.02$ ). In addition, the duration of vancomycin therapy attributable to physicians in the intervention group was $36 \%$ less than that of control physicians (26.5 vs 41.2 days; $P=.05$ ). Analysis of pharmacy data confirmed a decrease in the overall hospital use of intravenous vancomycin during the study period.

The authors concluded that implementation of computer-guided physician order entry decreased vancomycin use. Computerized guidelines represent a promising tool for changing prescribing practices.

FROM: Shojania KG, Yokoe D, Platt R, Fiskio J, Ma'luf $\mathrm{N}$, Bates DW. Reducing vancomycin use utilizing a computer guideline: results of a randomized controlled trial. $J$ Am Med Inform Assoc 1998;5:554-562.

\section{MRSA Bloodstream Infection and Mortality}

Moreira and coinvestigators from the Universidade Federal de São Paulo, Brazil, have reported a study to identify the attributed mortality rate of bloodstream hospital infection by methicillin-resistant Staphylococcus aureus (MRSA) and its effect on length of hospital stay. In a casecontrol study conducted in a 660-bed, tertiary-care teaching hospital in São Paulo, Brazil, 71 adult patients with hospitalacquired MRSA bacteremia diagnosed between January 1, 1991, and September 30, 1992, and 71 MRSA-free controls were matched on age, gender, underlying disease, surgical procedure, same risk time, and admission date.

MRSA accounted for $73 \%$ of hospital bloodstream infections involving Staphylococcus aureus. Mortality rates were $56 \%(40 / 71)$ for cases and $11 \%(8 / 71)$ for controls. The attributable mortality rate was $45 \%\left(\mathrm{OR}=17.0 ; \mathrm{CI}_{95}\right.$, $3.58-202.26 ; P=.000001)$. The median length of hospital stay was 32.6 days for the cases and 29.8 for the controls $(P=.32)$.

The authors concluded that a high proportion of
Staphylococcus aureus bacteremia involved MRSA. Nosocomial bloodstream infection with MRSA provides a high level of mortality independently from the patients' base disease, without increasing their hospital length of stay.

FROM: Moreira M, Medeiros EA, Pignatari AC, Wey SB, Cardo DM. The effect of nosocomial bloodstream infection by Staphylococcus aureus resistant to oxacillin on the mortality and the length of hospitalization. Rev Assoc Med Bras 1998;44:263-268.

\section{New Assay for Rapid Detection of MRSA}

A multiplex polymerase chain reaction (PCR), involving detection of the $m e c \mathrm{~A}$ and $f e m \mathrm{~B}$ genes, was combined with a novel immunoassay system capable of detecting specific PCR products. The resulting PCR-immunoassay was evaluated in comparison with conventional microbiological techniques used in the routine diagnostic laboratory for the rapid identification of methicillin-resistant Staphylococcus aureus (MRSA), either in pure culture or in overnight broth cultures obtained following enrichment of patient screening swabs. Among the 480 purified isolates of staphylococci and 246 enrichment broths examined, only one false-negative result was obtained by PCR, compared with 18 false-negative results obtained by conventional methodology and demonstrated by further conventional examination. Five demonstrable false-positive results were obtained by conventional methodology, compared with a possible 10 by the PCR-immunoassay, although it was not certain that these 10 PCR results were true false positives, as, by definition, MRSA could not be isolated by conventional methodology.

The results indicated that the routine diagnostic laboratory was encountering difficulties in identifying MRSA correctly and that the conventional microbiological techniques lacked sensitivity. Overall, the PCR technique was more accurate and sensitive than conventional methodology in detecting MRSA, and results were available within 24 hours of screening swabs arriving in the laboratory, compared with a minimum of 48 to 72 hours by conventional techniques. The immunoassay system added to the usefulness of the method by allowing the detection of specific PCR products within 5 minutes of completing the PCR, without the normal additional step of agarose gel electrophoresis.

FROM: Towner KJ, Talbot DC, Curran R, Webster CA, Humphreys H. Development and evaluation of a PCRbased immunoassay for the rapid detection of methicillinresistant Staphylococcus aureus. J Med Microbiol 1998; 47:607-613. 